\section{THE ORGANISATION}

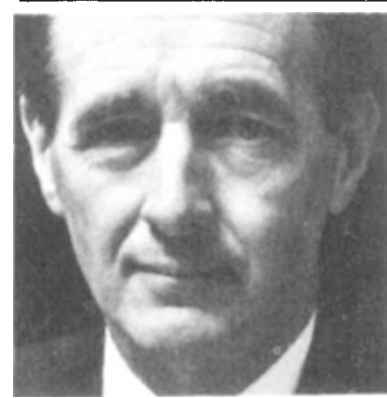

Who makes

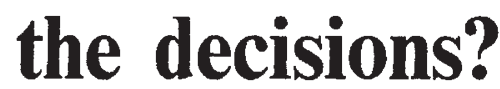

FINAL approval for ESA's scientific projects is granted by the Science Programme Committee (SPC), the body responsible to the ESA Council for running the science programme within the financial limits set by the Council. Each member state is represented on the SPC by its own delegates and is allowed one vote in any decision.

Projects for final selection are put to the SPC by the Director-General, $\mathrm{Mr}$ Roy Gibson. $\mathrm{He}$ is advised by the Science Advisory Committee (SAC) and four working groups: the astronomy working group (AWG), the solar system working group (SSWG), the materials sciences working group (MSWG) and the life sciences working group (LSWG). Experts appointed by the Director-General and acting in their own personal capacity, sit on each advisory body. The MSWG and LSWG were recently created. The two areas of science they serve are new to ESA. One life scientist, one meteorologist and four representatives of the astrophysical and geophysical sciences sit on the SAC. Its meetings are attended by the chairmen of the working groups.

The executive recommends that new project proposals are screened by the working groups. The money spent on early studies is authorised by the Director-General.

If preliminary studies are successful, the Director-General may recommend that a project be considered by the SPC. The SPC will then decide whether or not to go ahead. When a project has been approved, ESA puts out a call for proposals for experiments.

Although there is no formal link between the working groups and the SAC, in practice they work together quite closely. Typically, out of $10-20$ project proposals put before the working groups, 5-6 will be passed on to the SAC and three on to the SPC. On average the SPC chooses one major project for development every one and a half to two years.

So far, the SPC has only approved one experiment in the materials and life sciences, the Sled for Spacelab, to be funded out of the mandatory science budget. The rest of the experiments approved in these disciplines are for the first Spacelab payload and are being paid for in individual countries and out of the Spacelab budget.

As well as vetting individual project proposals, the working groups and the SAC discuss overall policy for the science programme. In 1976, for example, the AWG and SSWG both produced reports on the science and projects needed in their respective fields up to 1990. About once every four years, the SAC also discusses plans for the future, such as it did last week.

Responsibility for seeing that the science programme is put into practice rests with $\mathrm{Mr}$ Ernst Trendelenburg, Director of Scientific Programmes. His responsibility now includes future science programmes. To speed up decision-making, he would like to see the number of early studies cut, especially those for projects which are obviously unsuitable for further development from the outset.

\section{Four steps to get a project off the ground}

A SCIENTIFIC mission proposal which has been studied by the relevant working group and found scientifically worthy will go through four 'stages' before it is finally approved and the spacecraft is built. Each stage is independent of the others so that the mission can be scrapped at the end of any one without breaking contracts or jeopardising the money which would be spent in the subsequent stages. Most projects which go beyond phase $\mathrm{A}$, however, are completed. The steps in a mission's development are as follows:

Mission definition or assessment. This is a study stage to define the mission and its payload. It is done under the direction of ESA by seven to ten scientific experts in the mission's field of study. They measure the interest shown in the mission by the scientific community in Europe, assess its scientific soundness and the ability of European universities and institutes to build instruments capable of achieving its scientific objectives. They also predict what sort of system will evolve, how

- turn to next pag

\title{
The cost: where the money comes from, where it goes
}

ESA's TOTAL budget for 1978 is about $530 \mathrm{mau}$ (one mau is roughly US $\$ 1.3$ million). Of this, $75.5 \mathrm{mau}$ is to be spent on the science programme, about $80 \mathrm{mau}$ on the general budget and the rest on Spacelab, Earthnet and applications programmes such as telecommunications satellites, Meteosat and the development of ESA's own launcher, Ariane.

The science budget and part of the general budget are mandatory. Each member state contributes to them in proportion to its gross national product. Thus, in 1976, Belgium contributed $3.48 \%$, Denmark $2.26 \%$, France $21.20 \%$, Germany $25.25 \%$, Italy $13.23 \%$, the Netherlands $5.34 \%$, Spain $4.44 \%$, Sweden $4.46 \%$, Switzerland $3.56 \%$ and the United Kingdom $16.47 \%$. So far all ESA's scientific missions have been conducted through the mandatory programme, even though, in principle, nothing excludes a group of interested member states from funding an optional one.

The mandatory part of the general budget funds 'basic activities' such as the technological research programme at ESTEC, the salaries of some headquarter's staff, the documentation centre at Frascati and the infrastructure. In 1978 it amounted to about 54 mau. The rest of the general budget is for facilities which cannot be charged to any particular programme such as the cost of buying the agency's headquarters in Paris and the cost of running the Ariane's launch pad at Kouru in French Guiana.

All of the science budget is spent on building spacecraft and very large instruments such as those for Spacelab or large observatory-type free-flyers, and on maintaining facilities for tracking them and receiving data. Most of the experiments on board satellites, however, are built in universities and institutes and are funded out of national funds for scientific research.

In 1971 the mandatory science budget was fixed at $28 \mathrm{mau}$. In 1978 that level is $75.5 \mathrm{mau}$ and in 1979 it is expected to be about 79 mau. As scientific satellites cost between 50-100 mau, and some of them even more, this level of funding means that ESA can build one every one and a half to two years.

The capital cost of launches is not the only expense, and already the science budget is almost fully accounted for until the end of 1980 . It is proving difficult to find the $2 \mathrm{mau}$ needed to keep Cos B, ESA's cosmic ray satellite launched in 1975 , operating for another year. This sum has not already been included in next year's budget because Cos B has exceeded its expected lifetime. 
much it will cost and what sort of effort in terms of manpower and technological research will be needed. The study normally takes three months.

Phase A or study stage. Those proposals not thrown out after the assessment stage are subjected to a more detailed definition. This is usually done under the direction of a technical study team which may contract out to industry some parts of the study. It involves a detailed estimate of the cost and time to completion and a thorough description of the payload, spacecraft, orbit, ground support and data handling and analysis. Typically the study stage lasts nine months and costs $1 \%$ of the final project cost.

Phase $B$ or design stage. A scientific project which enters this stage has usually been approved for development by the SPC. It is put under the management of a project team which will be responsible for it until it has been launched and is operating. The project team places contracts in industry for the design of the spacecraft. Typically, phase B takes one year and costs $10 \%$ of the final cost of the project.

Phase C/D or development and production. Typically, for a scientific satellite, this will take three years and cost 40-70 mau.

The project teams include engineers from ESTEC's department of development and technology (DDT). When they are not part of a project team they do research in one of the DDT's divisions on various aspects of advanced space technology. This research is important for keeping Europe in the forefront of space technology.

Before launch all ESA satellites are tested at ESTEC. It has the only facility in Europe-a high vacuum chamber $14 \mathrm{~m}$ high and $10 \mathrm{~m}$ in diameter-large enough to test a satellite with its booms deployed or the nose cone of Ariane. Once the satellite is in orbit, however, operational responsibility for it shifts to the European Space Operations Centre (ESOC) at Darmstadt, West Germany. As well as control of the orbit, ESOC is responsible for setting up ground stations to receive data.

One of ESA's objectives is to encourage development of European industry. This is reflected in the way it puts out tenders to industry for its scientific satellites. Its industrial policy states that each member state should receive the same percentage of the total contract put out to tender as it contributes to the mandatory science budget. This is irrespective of whether it is participating in the scientific payload. This policy is controlled by the Industrial Policy Committee (IPC) which can suggest how contracts should be placed to ensure that each country gets a fair return.

ESA'S SCIENTIFIC RECORD

\section{The past ...}

ESA and its predecessor ESRO have launched twelve scientific satellites since the first launch in May 1968. Six of them are still operating: $\operatorname{Cos} \mathbf{B}$, a highly successful gamma ray observatory launched in April 1975; Geos 1, which failed to reach geostationary orbit in April 1977 but is producing some useful magnetospheric data; ISEE-2, which was launched in October 1977 and is observing the interaction of the solar wind and the Earth's magnetosphere in conjunction with NASA's ISEE-1; Meteosat, launched in November 1977, contributing to the Global Atmospheric Research Programme and the World Weather Watch; International Ultraviolet Explorer, the ultraviolet astronomical observatory, launched as a NASA-UK-ESA collaboration in January 1978; and Geos 2, a 'copy' of Geos 1, successfully launched into geostationary orbit in June 1978 to observe the magnetosphere.

Of the previous seven satellites, five were designed for magnetospheric and atmospheric experiments, and two for high energy astronomy: ESRO-II for cosmic rays and solar X-rays, launched May 1968) and TD-1 (a UV satellite launched March 1972 and operational for two years but troubled by malfunctioning tape recorders). Of the complete list of thirteen satellites, nine were for Earth-related observations (mostly on Sun-Earth interaction) and four for astronomy. Astronomical satellites were launched in 1968, 1972, 1975 and 1978.

\section{The present ... .}

ESA's report to the 21st COSPAR meeting in June 1978 says that while Sun-Earth relationships "continue to be an important feature of the agency's scientific programme", there is a change afoot: "an increased emphasis is being placed on astronomical studies". This is reflected to some extent in the current list of approvals (four main projects, two of which are astronomical), and to a greater extent among the competing proposals for the next project (three out of five are for astronomy). Approved are:

The first Spacelab payload. Spacelab is scheduled to fly with the tenth flight of the Space Shuttle on 3 December 1983. It will be manned, but will stay aloft for only 7-30 days. According to ESA's 1978 report to COSPAR "the composition of a typical Spacelab payload will be distinctly different from that of a conventional research satellite". Life sciences and materials sciences will benefit from 'look-see' experiments in a new environment. But the observing time is too short for much astronomy.

EXOSAT. This $0.1 \mathrm{keV}$ to $50 \mathrm{keV}$ $\mathrm{X}$-ray astronomy satellite is scheduled for launch on Ariane in February or March 1981 (or by Thor-Delta if Ariane is not ready). It has two operating modes: occultation, using the Earth or Moon to determine the position and structure of X-ray sources, and arbitrary pointing, which will be used to determine this spectral and temporal variation.

Out of Ecliptic/Solar Polar Mission. This is a joint mission with NASA to launch two spacecraft in February 1983 , to fly out of the plane of planetary orbits and around the North and South poles of the Sun. The gravitational field of Jupiter will be used to swing the spacecraft out of the ecliptic. The objective is to extend into three dimensions our knowledge of the solar corona, the solar wind, the solar magnetic field, the cosmic ray flux, and interplanetary/interstellar neutral gas and dust.

The space telescope. This, as ESA's latest report to COSPAR says, "is the most ambitious project in space astronomy currently planned and will dominate astronomical research for the rest of the century". ESA is participating at a level that will allow Europe's astronomers just $15 \%$ of the observing time on the high-resolution, 2.4 metre telescope. The telescope should be launched from the space shuttle at the end of 1983 with five separate focal plane instruments. In a $500-\mathrm{km}$ high orbit, it will be retrievable and refurbishable, and should serve astronomers for two decades.

\section{The future ...}

THE European Space Research and Technology Centre (ESTEC) in the Netherlands is investigating the scientific and technical merit of several spacecraft which will compete for selection as the scientific project to follow the ESA/NASA space telescope which is due for launch in 1983.

To effectively use the science budget ESA's Science Policy Committee (SPC) will have to decide on which experiment to launch by the beginning of 1980 . The next mission should then be ready for launch in 1985 .

The options are already known. According to Dr D. E. Page, head of the science department at ESTEC, any new mission proposals could not be studied in time for next year's decision 